\title{
Dipolar field effects on the critical current for spin transfer switch of iron and permalloy nanoelements
}

L. L. Oliveira, J. T. S. Dantas, R. M. Souza, A. S. Carriço, and Ana L. Dantas

Citation: Journal of Applied Physics 115, 17D130 (2014);

View online: https://doi.org/10.1063/1.4865317

View Table of Contents: http://aip.scitation.org/toc/jap/115/17

Published by the American Institute of Physics

\section{Articles you may be interested in}

Controlling the vortex core of thin Permalloy nano-cylinders dipolar coupled to Co polarizers Journal of Applied Physics 115, 17D110 (2014); 10.1063/1.4860056

Ferromagnetic resonance of compensated ferromagnetic/antiferromagnetic bilayers Journal of Applied Physics 112, 073907 (2012); 10.1063/1.4757032

Controlling the core-to-core distance of vortex pairs in exchange-biased iron elliptical nanoelements Journal of Applied Physics 111, 07D102 (2012); 10.1063/1.3670978

Nucleation of vortex pairs in exchange biased nanoelements

Journal of Applied Physics 109, 07D314 (2011); 10.1063/1.3537924

\section{Scilight} Sharp, quick summaries illuminating the latest physics research

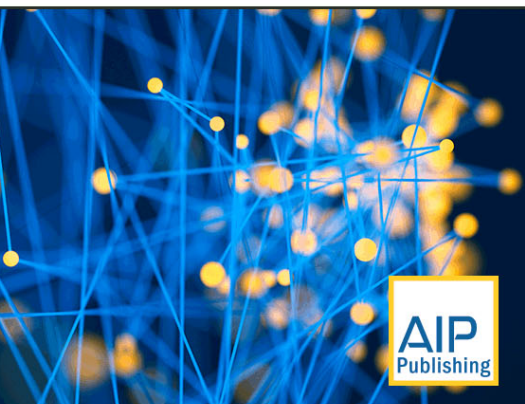




\title{
Dipolar field effects on the critical current for spin transfer switch of iron and permalloy nanoelements
}

\author{
L. L. Oliveira, ${ }^{1}$ J. T. S. Dantas, ${ }^{1}$ R. M. Souza, ${ }^{1}$ A. S. Carriço, ${ }^{1, a)}$ and Ana L. Dantas ${ }^{2}$ \\ ${ }^{1}$ Departamento de Física, Universidade Federal do Rio Grande do Norte, 59072-970 Natal, RN, Brazil \\ ${ }^{2}$ Departamento de Física, Universidade do Estado do Rio Grande do Norte, 59610-210 Mossoró, RN, Brazil
}

(Presented 7 November 2013; received 23 September 2013; accepted 7 November 2013; published online 10 February 2014)

\begin{abstract}
We report a theoretical study of dipolar effects on the switching current density of soft ferromagnetic elliptical nanoelements. Relevant changes on the critical current value are found according to the orientation of the magnetization and the spin polarization with the major axis. We show that the critical current density may be reduced by as much as $92 \%$ for thin nanoelements magnetized along the minor axis direction, using in-plane spin polarization parallel to the magnetization. (C) 2014 AIP Publishing LLC. [http://dx.doi.org/10.1063/1.4865317]
\end{abstract}

Spin-transfer torque (STT) magnetization switching in submicron sized magnetic systems has recently received considerable attention, both for its fundamental interest, as a new spintronic effect, as well as for its large potential for the development of new spintronic devices. It was theoretically proposed by Slonczewski ${ }^{1}$ and Berger $^{2}$ as a new mechanism to locally manipulate the magnetic order of nano-sized systems, and led to an ever increasing interest in the small-scale architecture of the magnetic order of having magnetic nanoelements.

One promising application of STT magnetization switching is the new generation of nonvolatile random access memories (MRAM) based on magnetic tunnel junction cells. Recent efforts to reduce the switching current density of STT-MRAM cells have focused on the optimization of the magnetic properties of the switching layer, ${ }^{3-5}$ the use of composite free layer structures, ${ }^{6-8}$ and the use of hard axis external field. ${ }^{9}$ We presently report a discussion of another possible way to achieving the reduction of the switching current density.

The magnetic stability of soft ferromagnetic nanoelements with sub-micrometer dimensions is largely due to the shape anisotropy. This opens a promising route to tailoring the spin polarized current effects on the magnetic order of MRAM cells. The ground state of elliptical nanoelements corresponds to magnetization along the major axis. Thus, the switching current density for magnetization along the major axis is expected to be larger than the corresponding value of a circular nanoelement. Also, if the magnetization is along the minor axis direction, smaller external effects, either external field or spin polarized electrical current, are required for switching. Thus, provided the thermal stability is not compromised, one may seek low switching current density systems for device applications in this configuration.

In this paper, we report a theoretical investigation of the impact of the dipolar field on the critical current for switching elliptical $\mathrm{Fe}$ and $\mathrm{Py}^{\mathrm{TM}}$ thin nanoelements.

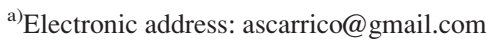

We use a generalized Landau-Lifshitz-GilbertSlonczewski (LLGS) equation $d \vec{M} / d t=-\gamma^{\prime} \vec{M} \times \vec{H}^{*}$, with $\vec{H}^{*}$ given by

$$
\begin{aligned}
\vec{H}^{*}= & \vec{H}_{e f f}+\alpha \hat{m} \times \vec{H}_{e f f}+\frac{2 \mu_{B} g J}{\left(1+\alpha^{2}\right) e t M_{S}}(\hat{m} \times \hat{p}) \\
& -\frac{2 \mu_{B} g \alpha J}{\left(1+\alpha^{2}\right) e t M_{S}} \hat{p} .
\end{aligned}
$$

The magnetization dynamics includes the effect of damping, the contribution of the Slonczewski spin transfer torque, ${ }^{1}$ and the conservative part of the magnetic energy, represented by the effective field $\vec{H}_{e f f} \cdot \vec{M}$ is the magnetization, $\hat{m}=\vec{M} / M_{S}$, and $M_{S}$ is the saturation magnetization. $g$ is a function of the orientation of the magnetization relative to the spin polarization direction ${ }^{1}$ and is given by

$$
g=\left[-4+(1+\eta)^{3} \frac{(3+\hat{m} \cdot \hat{p})}{4 \eta^{3}}\right]^{-1} .
$$

$J$ is the current density, $t$ is the thickness of the nanoelement, and $e$ is the absolute value of the electron charge. $\hat{p}$ is the electron spin polarization direction, $\gamma^{\prime}=\gamma /\left(1+\alpha^{2}\right), \gamma$ is the electron gyromagnetic ratio, $\alpha$ is the Gilbert damping parameter, and $\eta$ is the spin polarization factor. We use $\alpha=0.4$ and $\eta=0.4$.

The z-axis is chosen perpendicular to the surface of the nanoelement and the uniaxial anisotropy easy axis is inplane along the $\hat{e}_{A}$ direction. We use cubic simulation cells with edge of $d=2 \mathrm{~nm}$. The effective field is given by $\vec{H}_{\text {eff }}$ $=-(\partial E / \partial \vec{M})$ and the energy density is given by

$$
\begin{aligned}
E= & \frac{A}{d^{2}} \sum_{j} \sum_{k}\left(1-\hat{m}_{j} \cdot \hat{m}_{k}\right)-K \sum_{j}\left(\hat{m}_{j} \cdot \hat{e}_{A}\right)^{2} \\
& +\frac{M_{S}^{2}}{2} \sum_{j} \sum_{k}\left(\frac{\hat{m}_{j} \cdot \hat{m}_{k}}{n_{j k}^{3}}-\frac{3\left(\hat{m}_{j} \cdot \hat{n}_{j k}\right)\left(\hat{m}_{k} \cdot \hat{n}_{j k}\right)}{n_{j k}^{5}}\right),
\end{aligned}
$$

where the first term is the exchange energy, coupling nearest-neighbor magnetic moments, and $A$ is the ferromagnetic exchange stiffness. The second term is the anisotropy 


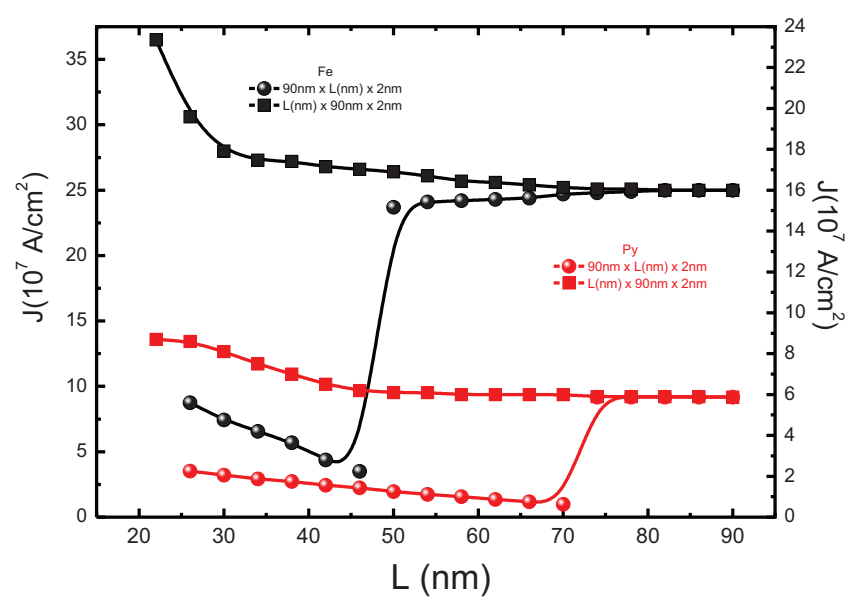

FIG. 1. Switching current densities of iron (left hand side axis) and $\mathrm{Py}^{\mathrm{TM}}$ (right hand side axis) elliptical nanoelements: square symbol curves for magnetization along the major axis and circular symbol curves for magnetization along the minor axis.

energy and the last term involves an unrestricted sum of the magnetostatic energy over all pairs of cells. $\hat{m}_{i}$ is the direction of the magnetic moment of the $i$ th cell, and $n_{i j}$ is the distance between the cells $i$ and $j$ in units of cell size $d$. The current density is perpendicular to the surface of the nanoelement and the spin polarization is in the $x y$-plane.

The magnetic structure of the stationary state $(d M / d t=0)$ is found by seeking a set of directions of the moments in all cells $\left(\hat{m}_{i}, i=1, \ldots, N\right)$ which makes the effective torque $\left(\gamma^{\prime} \vec{M} \times \vec{H}^{*}\right)$ in each one of them smaller than $10^{-14} \mathrm{Am}^{2} / \mathrm{s}$. The switching curve is obtained by starting with $J=0$ in a nearly uniform state, either along the minor-axis direction or the major-axis direction, and finding the magnetic structure of the stationary state which accommodates the competing trends of the exchange and dipolar energies to the geometrical confinement of the magnetic structure in the nanoelement. For each value of the current density thereafter, the selfconsistent search ${ }^{10}$ for the stationary state magnetic structure is initialized with the magnetic phase corresponding to the previous value of the current density.

$\mathrm{Fe}$ has larger values of the saturation magnetization and uniaxial anisotropy energy. Therefore for nanoelements with the same dimensions, the critical current of Fe nanoelements is larger. As shown in Fig. 1, the critical currents for circular $(90 \mathrm{~nm} \times 90 \mathrm{~nm} \times 2 \mathrm{~nm})$ and nearly circular Fe nanoelements are around $25 \times 10^{7} \mathrm{~A} / \mathrm{cm}^{2}$, while for $\mathrm{Py}^{\mathrm{TM}}$ nanoelements the corresponding values are around $6 \times 10^{7} \mathrm{~A} / \mathrm{cm}^{2}$.

In Fig. 1, we also show the values of the switching current density for $90 \mathrm{~nm} \times L(\mathrm{~nm}) \times 2 \mathrm{~nm}$ nanoelements magnetized along the minor axis, and for $L(\mathrm{~nm}) \times 90 \mathrm{~nm} \times 2 \mathrm{~nm}$ nanoelements magnetized along the major axis. In both cases, we use the uniaxial anisotropy and the current spin polarization parallel to the magnetization.

For magnetization along the minor axis, the $\mathrm{Py}^{\mathrm{TM}}$ $90 \mathrm{~nm} \times 70 \mathrm{~nm} \times 2 \mathrm{~nm}$ and the Fe $90 \mathrm{~nm} \times 46 \mathrm{~nm} \times 2 \mathrm{~nm}$ nanoelements have critical current densities of $6 \times 10^{6} \mathrm{~A} / \mathrm{cm}^{2}$ and $3 \times 10^{7} \mathrm{~A} / \mathrm{cm}^{2}$, corresponding to reductions of $90 \%$ and $86 \%$ on the critical current densities of the corresponding circular nanoelements.
TABLE I. Critical current density $\left(J_{C}\left(10^{7} \mathrm{~A} / \mathrm{cm}^{2}\right)\right)$.

\begin{tabular}{lcccccccc}
\hline \hline & \multicolumn{2}{c}{ Circular } & & \multicolumn{2}{c}{$2 a=150 \mathrm{~nm}^{\mathrm{a}}$} & & \multicolumn{2}{c}{$a<r^{\mathrm{b}}$} \\
\cline { 2 - 3 } \cline { 8 - 9 } Diameter $2 r(\mathrm{~nm})$ & $\mathrm{Fe}$ & $\mathrm{Py}^{\mathrm{TM}}$ & & $\mathrm{Fe}$ & $\mathrm{Py}^{\mathrm{TM}}$ & & $\mathrm{Fe}$ & $\mathrm{Py}^{\mathrm{TM}}$ \\
\hline 62 & 25 & 5.9 & 27.0 & 8.3 & & $2.2(38 \mathrm{~nm})^{\mathrm{c}}$ & $0.8(50 \mathrm{~nm})^{\mathrm{c}}$ \\
66 & 25 & 5.9 & 26.2 & 8.0 & & $2.9(38 \mathrm{~nm})^{\mathrm{c}}$ & $0.7(54 \mathrm{~nm})^{\mathrm{c}}$ \\
70 & 25 & 5.9 & & 26.4 & 7.9 & & $2.0(42 \mathrm{~nm})^{\mathrm{c}}$ & $0.6(58 \mathrm{~nm})^{\mathrm{c}}$ \\
90 & 25 & 5.9 & & 25.4 & 7.6 & & $3.0(46 \mathrm{~nm})^{\mathrm{c}}$ & $0.6(70 \mathrm{~nm})$ \\
110 & 24.9 & 5.9 & 24.9 & 7.4 & & $3.0(54 \mathrm{~nm})^{\mathrm{c}}$ & $0.7(78 \mathrm{~nm})^{\mathrm{c}}$ \\
\hline \hline
\end{tabular}

${ }^{a}$ Magnetization along the major axis.

${ }^{\mathrm{b}}$ Magnetization along the minor axis.

${ }^{\mathrm{c}}$ The minor axis length.

For magnetization along the major axis, the $\mathrm{Py}^{\mathrm{TM}}$ $20 \mathrm{~nm} \times 90 \mathrm{~m} \times 2 \mathrm{~nm}$ and the Fe $20 \mathrm{~nm} \times 90 \mathrm{~nm} \times 2 \mathrm{~nm}$ nanoelements have critical current densities of $8.65 \times 10^{7} \mathrm{~A} / \mathrm{cm}^{2}$ and $36.6 \times 10^{7} \mathrm{~A} / \mathrm{cm}^{2}$, corresponding to an increase in $44 \%$ compared with the critical current densities of the corresponding circular nanoelements.

The leading features of the results shown in Fig. 1 are summarized in Table I for nanoelements of dimensions ranging from $62 \mathrm{~nm}$ up to $110 \mathrm{~nm}$.

Notice that for magnetization along the minor axis, the Fe $42 \mathrm{~nm} \times 70 \mathrm{~nm} \times 2 \mathrm{~nm}$ nanoelement switches at a current density of $2 \times 10^{7} \mathrm{~A} / \mathrm{cm}^{2}$, with a $92 \%$ reduction of the critical current density $\left(25 \times 10^{7} \mathrm{~A} / \mathrm{cm}^{2}\right)$ of the circular nanoelement. Similarly, the $58 \mathrm{~nm} \times 70 \mathrm{~nm} \times 2 \mathrm{~nm} \mathrm{Py}{ }^{\mathrm{TM}}$ nanoelement switches at a current density of $0.6 \times 10^{7} \mathrm{~A} / \mathrm{cm}^{2}$, with a $90 \%$ reduction of the critical current density $\left(5.9 \times 10^{7} \mathrm{~A} / \mathrm{cm}^{2}\right)$ of the circular nanoelement.

In Fig. 2, we show the switching curves of $\mathrm{Fe}$ $L(\mathrm{~nm}) \times 70 \mathrm{~nm} \times 2 \mathrm{~nm}$ nanoelements. In the inset, we show similar results for $\mathrm{Py}^{\mathrm{TM}}$ nanoelements. There are two regimes. Circular and nearly circular $\mathrm{Fe}$ nanoelements switch at current densities around $25 \times 10^{7} \mathrm{~A} / \mathrm{cm}^{2}$, while low aspect ratio $\mathrm{Fe}$ nanoelements switch at current densities smaller than $5 \times 10^{7} \mathrm{~A} / \mathrm{cm}^{2}$.

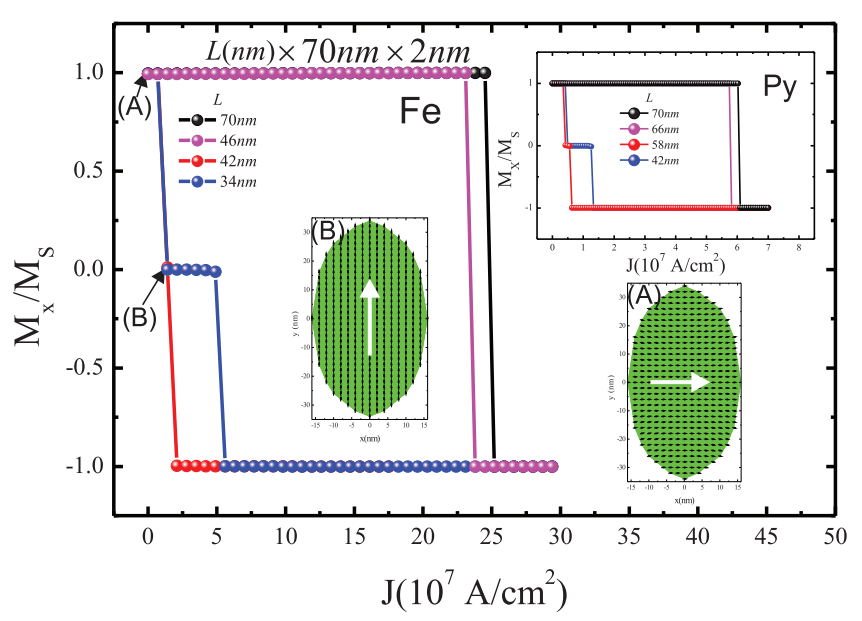

FIG. 2. Switching curves of Fe and $\mathrm{Py}^{\mathrm{TM}}$ (inset) elliptical L $(\mathrm{nm}) \times 70 \mathrm{~nm}$ $\times 2 \mathrm{~nm}$ nanoelements. The panels (A) and (B) show the magnetic structure at selected points in the curves. 

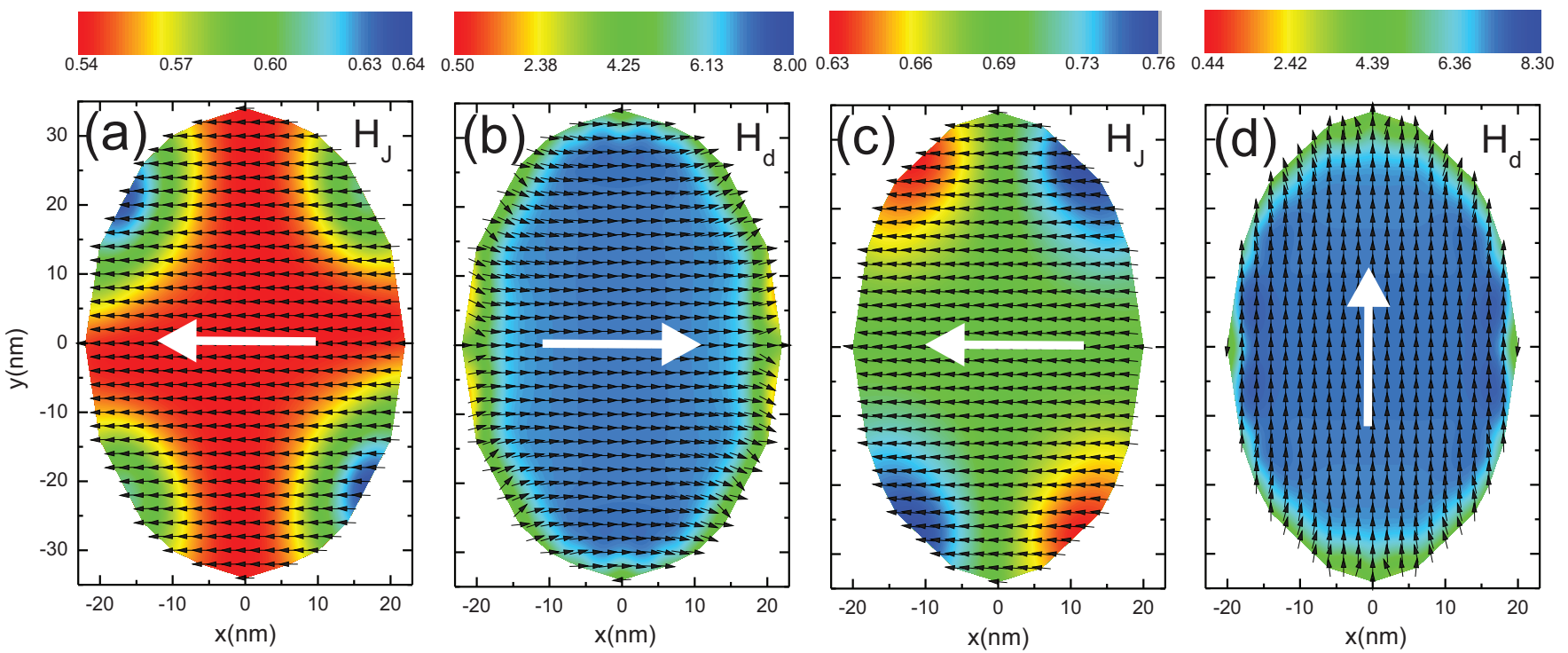

FIG. 3. Maps of the spin polarized current effective field (HJ) and of the dipolar field (Hd) at the switching point. (a) and (b) for Fe $46 \mathrm{~nm} \times 70 \mathrm{~nm} \times 2 \mathrm{~nm}$, and (c) and (d) for the Fe $42 \mathrm{~nm} \times 70 \mathrm{~nm} \times 2 \mathrm{~nm}$. The color bar code indicates the field intensity.

The low current density regime corresponds to nanoelements with minor axis length below a certain threshold value, leading to a reduced stability of the state with the magnetization along the hard axis of the dipolar field. The reversal is a two stage process. At a current density around $2 \times 10^{7} \mathrm{~A} / \mathrm{cm}^{2}$, the magnetization switches to the easy axis direction of the dipolar field. By further increasing the current density, the magnetization switches to the direction determined by the electron spin polarization.

In Fig. 3, we show the maps of the spin polarized current effective field $\left(H_{J}\right)$ and of the dipolar field $\left(H_{d}\right)$ at the switching point. Figs. 3(a) and 3(b) concern the switching of the Fe $46 \mathrm{~nm} \times 70 \mathrm{~nm} \times 2 \mathrm{~nm}$ nanoelement at a current density of $23 \times 10^{7} \mathrm{~A} / \mathrm{cm}^{2}$. Figs. 3(c) and 3(d) are for the switching of the $\mathrm{Fe} 42 \mathrm{~nm} \times 70 \mathrm{~nm} \times 2 \mathrm{~nm}$ nanoelement at a rather smaller current density of $1.4 \times 10^{7} \mathrm{~A} / \mathrm{cm}^{2}$. See that the dipolar field intensities have nearly equal values. However, the relative orientation of the effective current field and the dipolar field favors the low switching current in the nanoelement with small aspect ratio.

Notice that the threshold value of the minor axis length for the Fe nanoelement corresponds to an aspect ratio of 0.6, while for the $\mathrm{Py}^{\mathrm{TM}}$ nanoelement it is larger (0.83). This extra stability of magnetic phase of the $\mathrm{Fe}$ nanoelements magnetized along the minor axis direction is due to the uniaxial anisotropy. $\mathrm{Py}^{\mathrm{TM}}$ is isotropic. Thus, the dipolar effects dragging the minor axis uniform state (along the hard axis of the shape anisotropy) to the easy axis direction occur for larger aspect ratio values.

In summary, we have shown that the shape anisotropy of $\mathrm{Fe}$ and $\mathrm{Py}^{\mathrm{TM}}$ nano-ellipses may be explored to design systems with considerable reduction, by as much as $92 \%$, of the switching current.

The authors acknowledge financial support from CNPq, CAPES, and FAPERN. The work of A.S.C. was supported by CNPq Grant No. 350773 and the work of A.L.D. was supported by CNPq Grant No. 309676.

${ }^{1}$ J. C. Slonczewski, J. Magn. Magn. Mater. 159, L1 (1996).

${ }^{2}$ L. Berger, Phys. Rev. B 54, 9353 (1996).

${ }^{3}$ M. T. Rahman, A. Lyle, P. Khalili Amiri, J. Harms, B. Glass1, H. Zhao, G. Rowlands, J. A. Katine, J. Langer, I. N. Krivorotov, K. L. Wang, and J. P. Wang, J. Appl. Phys. 111, 07C907 (2012).

${ }^{4}$ K. Oguz, M. Ozdemir, O. Dur, and J. M. D. Coey, J. Appl. Phys. 111, 113904 (2012).

${ }^{5}$ K. Yagami, A. A. Tulapurkar, A. Fukushima, and Y. Suzuki, Appl. Phys. Lett. 85, 5634 (2004).

${ }^{6}$ K. Lee, W.-C. Chen, X. Zhu, X. Li, and S.-H. Kang, J. Appl. Phys. 106, 024513 (2009).

${ }^{7}$ J. Hayakawa, S. Ikeda, Y. M. Lee, R. Sasaki, T. Meguro, F. Matsukura, H. Takahashi, and H. Ohno, Jpn. J. Appl. Phys. Part 2 45, L1057 (2006).

${ }^{8}$ M. Ichimura, T. Hamada, H. Imamura, S. Takahashi, and S. Maekawa, J. Appl. Phys. 105, 07D120 (2009).

${ }^{9}$ T. Devolder, P. Crozat, J.-V. Kim, C. Chappert, K. Ito, J. A. Katine, and M. J. Carey, Appl. Phys. Lett. 88, 152502 (2006).

${ }^{10}$ A. S. M. Silva, A. L. Dantas, G. O. G. Rebouças, and A. S. Carriço, J. Appl. Phys. 109, 07D314 (2011). 